\title{
Lactation Suppression Treatments
}

\section{ART ICLE INF O}

\section{Article Type}

Analytical Review

\section{Authors}

Hashemi Jam M.S.* MD,

Roustaei Z. ${ }^{1} \mathrm{PhD}$

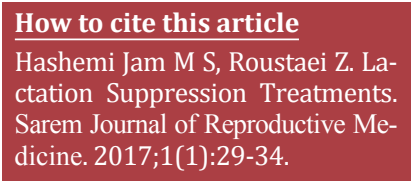

*Sarem Fertility \& Infertility Research Center (SAFIR), Sarem Women's Hospital, Tehran, Iran

${ }^{1}$ Sarem Fertility \& Infertility Research Center (SAFIR), Sarem Women's Hospital, Tehran, Iran

\section{Correspondence}

Address: Sarem Women's Hospital, Basij Square, Phase 3, Ekbatan Town, Tehran, Iran. Postal Code: 1396956111

Phone: +98 (21) 44670888

Fax: +98 (21) 44670432

dr.msdjam@gmail.com

\section{Article History}

Received: August 18, 2015

Accepted:December 24,2015

ePublished: February 15, 2017

\section{A B S T R A C T}

Introduction One of the most important changes occurs in the body of a pregnant woman is postpartum milk secretion. If the baby is not fed from this milk or the milk is not drained from the mother's breast in any other manner, some maternal problems will be arised. There are numerous drug and non-drug interventions to suppress lactation after childbirth and relief of its symptoms in women. This review report aimed to investigate the literature involved in this field to offer a single protocol in such cases. In the search strategy, we used the keywords of suppression of lactation, Treatments for suppression of lactation, Drugs in breastfeeding, Lactation after Perinatal, Neonatal, or Infant Loss by referring to the valid scientific databases, including Sciencedirect, Pubmed, Elsevier, Cochrane, and about 26 relevant papers were received and analyzed.

Conclusion Currently, the recommended methods for the cessation of lactation in women with necessary indications are not conclusive for all involved women. Identification and applying different methods to suppress lactation in mothers who have been banned to use milk for various reasons, can significantly help them to better manage suppression of lactation. Based on the relevant systematic review study, it seems that in case of low maternal milk secretion, it is better to use non-pharmacological methods to suppress lactation because of their fewer side effects; but the most effective treatments are the use of Bromocriptine and Cabergoline. But still the most common treatment for the suppression of lactation is administration of Estrasiol.

Keywords Suppression of Lactation; Breastfeeding; Limiting Breastfeeding

\section{I T A T I O N L I N K S}

[1] A retrospective drug use evaluation of cabergoline for lactation inhibition at a tertiary care teaching hospital in Qatar [2] Role of newer drug cabergoline in lactation suppression as compared to estrogen-androgen combination [3] Breastfeeding and maternal medications [4] Rationale and design of a randomized, controlled multicentre clinical trial to evaluate the effect of bromocriptine on left ventricular function in women with peripartum cardiomyopathy [5] Treatments for suppression of lactation [6] Physical mechanisms underlying neurite outgrowth: A quantitative analysis of neuronal shape [7] A Study of the association between drug abuse and duration of exclusive breastfeeding in mothers in Sabzevar City, Iran [8] Handbook on drug dependence management in pregnancy, childbirth, lactation and infancy [9] Alcohol during pregnancy and lactation: Recommendations versus real intake [10] Effects of alcohol consumption during pregnancy and/or lactation on the morphology of thyroid gland in male Wistar rat offspring [11] Alcohol consumption during pregnancy and breast feeding in Canada is prevalent and not strongly associated with mental health status [12] Investigation of the damaging effects of alcohol consumption during lactation on cerebellum structure and balance of neonates [13] When breast-feeding is not contraindicated: Do you know when to stop breast-feeding? [14] Determinants of breastfeeding initiation and cessation among employed mothers: A prospective cohort study [15] Determination of effective factors in breast feeding continuity for infants less than 1 year old in urban area of Bushehr Province [16] Exclusive breastfeeding among city-dwelling professional working mothers in Ghana [17] A survey on some effective factors on the duration of breastfeeding using survival analysis (Mazandaran province) [18] An exploration of the maternal experiences of breast engorgement and milk leakage after perinatal loss [19] Lactation after perinatal, neonatal, or infant loss [20] Pharmacological lactation suppression with D2 receptor agonists and risk of postpartum psychosis: A systematic review [21] An exploration of the experiences of mothers as they suppress lactation following late miscarriage, stillbirth or neonatal death. which citty? [22] Treatment for lactation suppression: Little progress in one hundred years [23] Complementary and alternative medicine in obstetrics [24] The effectiveness of stilboestrol in the suppression of postpartum lactation [25] Inhibition of lactation 
موارد منع شيردهى: علىرغم مزاياى بسيارى كه تغذيه با شير مادر

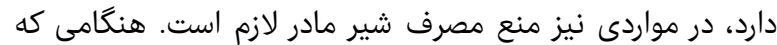

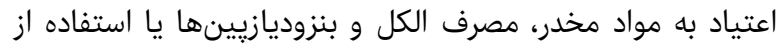

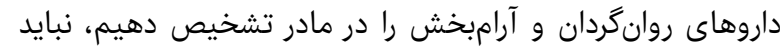

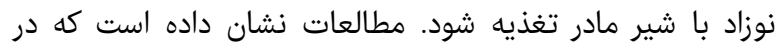

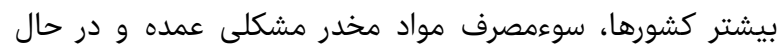

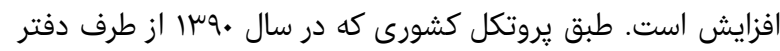

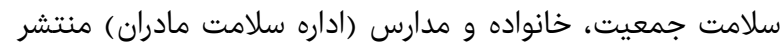

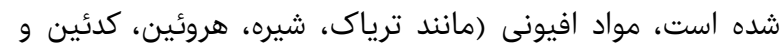

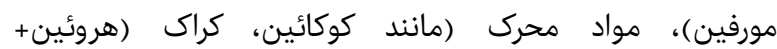

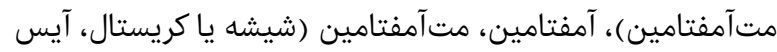

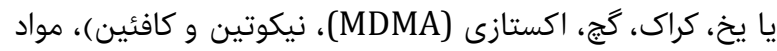

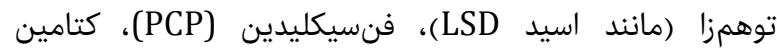

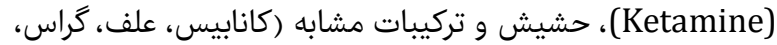

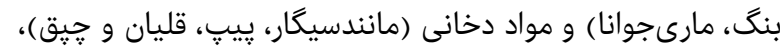

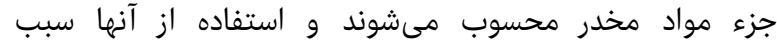

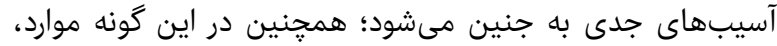

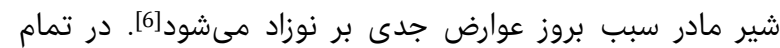

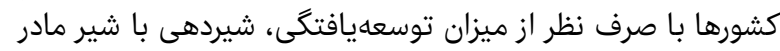

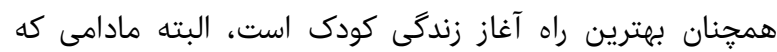

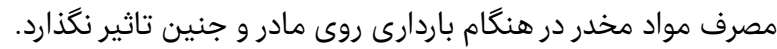

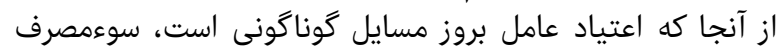

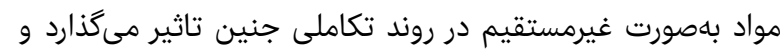

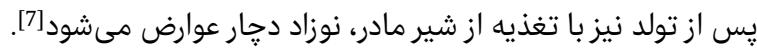

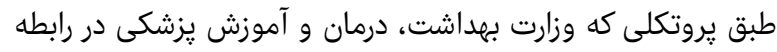

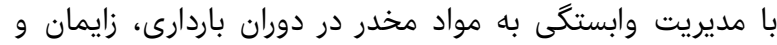

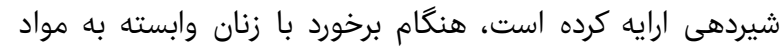

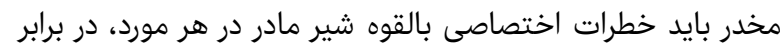

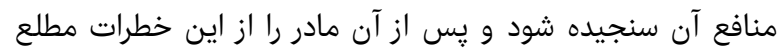

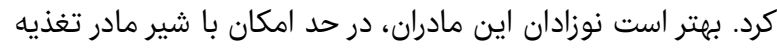

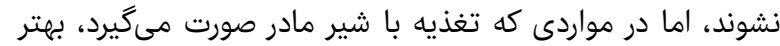

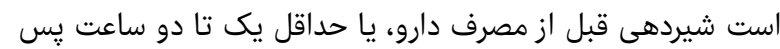

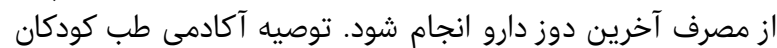

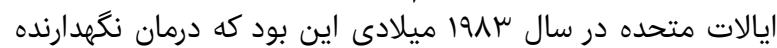

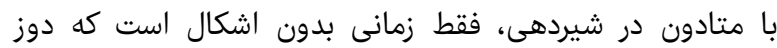

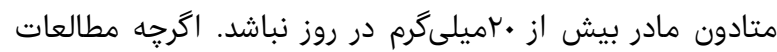

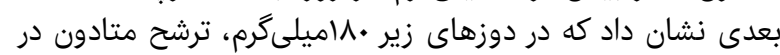

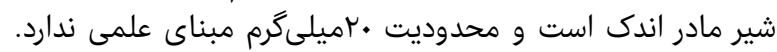

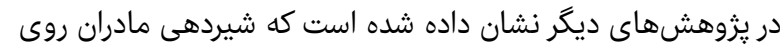

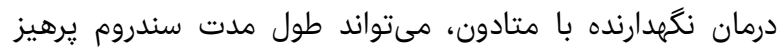

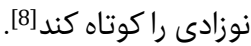

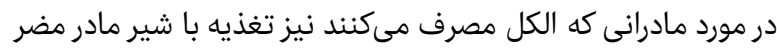

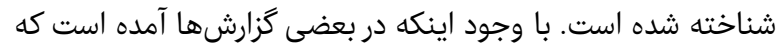

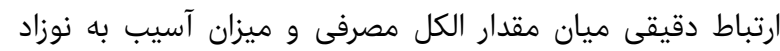

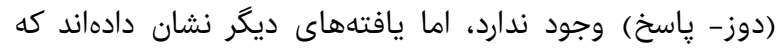

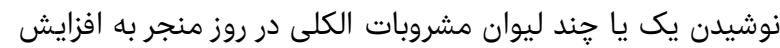

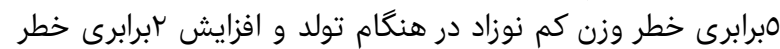

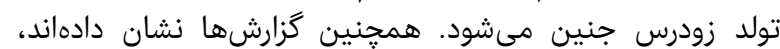

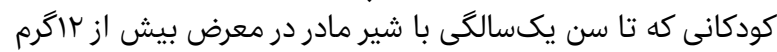

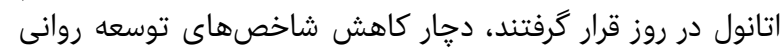

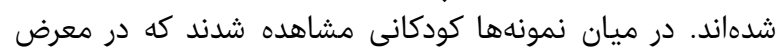

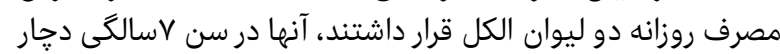

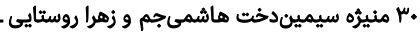

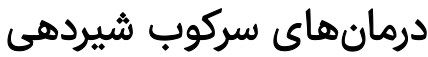

MD منيزه سيميندخت هاشمىجم مركز تحقيقات بارورى و نابارورى صارم، بيماردمارستان فوق تخصصى صارم، تهران،

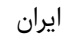

زهرا روستايى PhD مركز تحقيقات بارورى و نابارورى صارم، بيمارستان فوق تخصصى صارم، تهران، ايران

جكيده

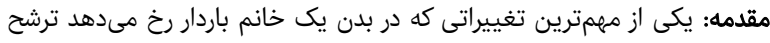

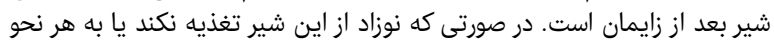

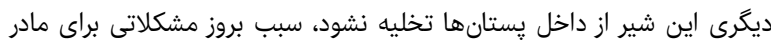

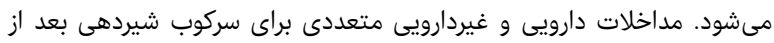

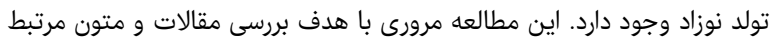

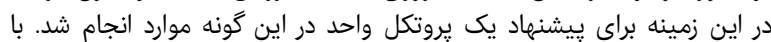

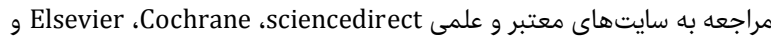

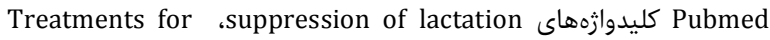
Lactation after ،Drugs in breastfeeding ،suppression of lactation Neonatal, or Infant Loss ،Perinatal

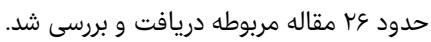

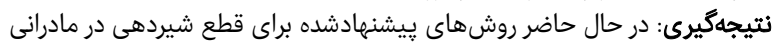

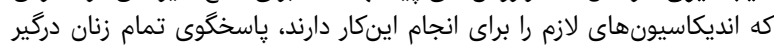

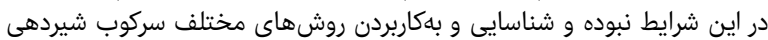

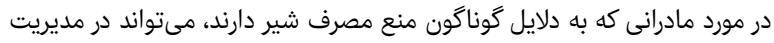

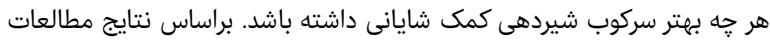

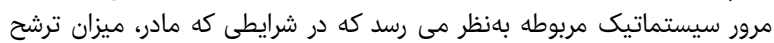

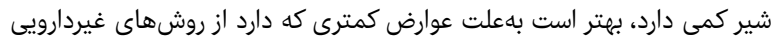

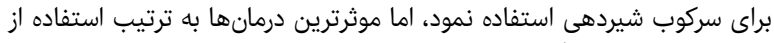

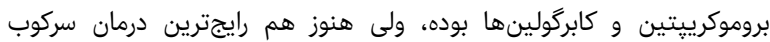
شيردهى از طريق استراديول است.

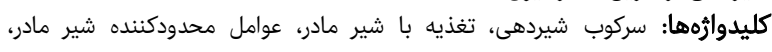

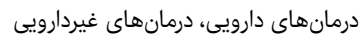

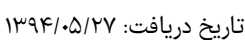

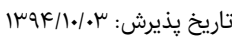

dr.msdjam@gmail.com :نويسنده مسئول

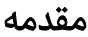

شيردهى يك فرآيند طبيعى و فيزيولوزيك يس از زايمان است.

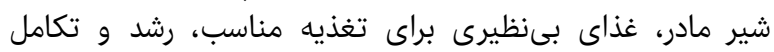

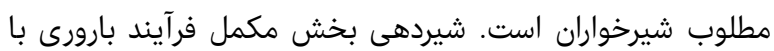

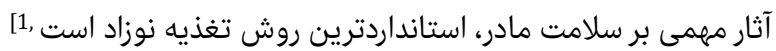

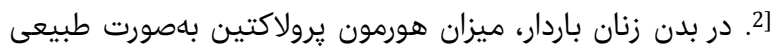

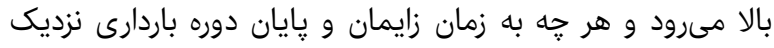

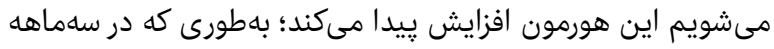

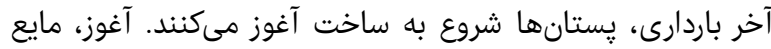

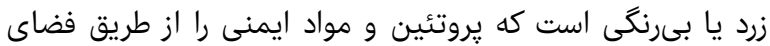

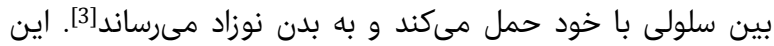

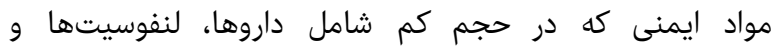

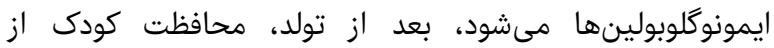

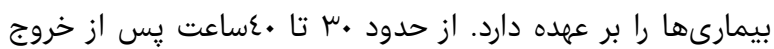

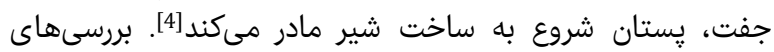

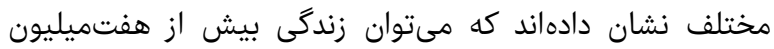

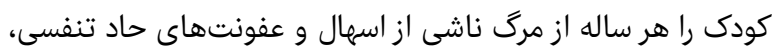

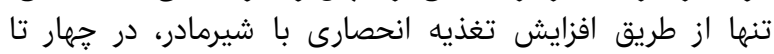

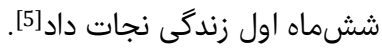




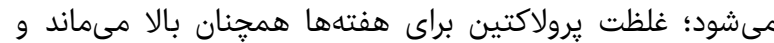

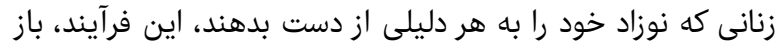

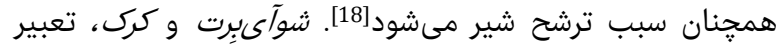

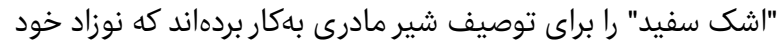

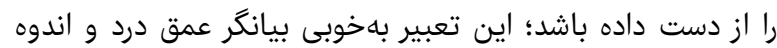

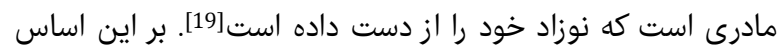

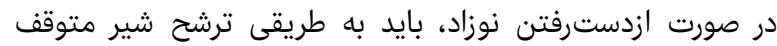

شود.

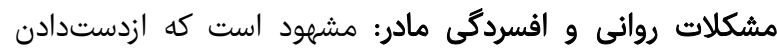

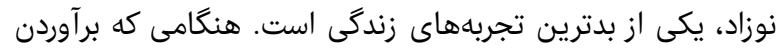

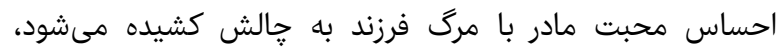

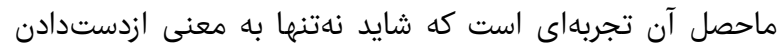

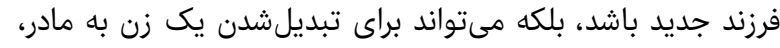

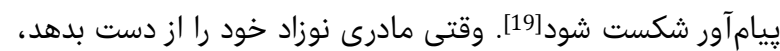

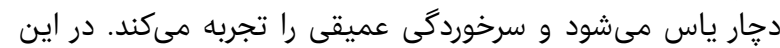

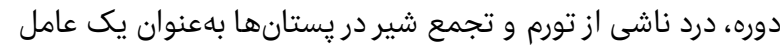

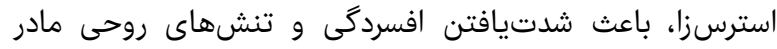

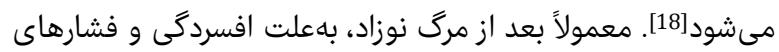

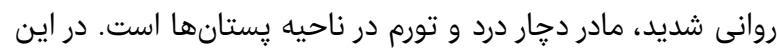

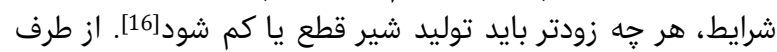

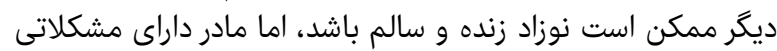

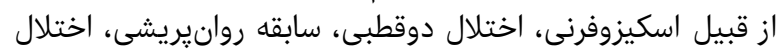

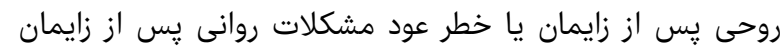

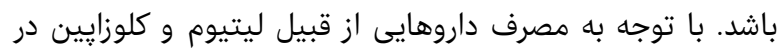

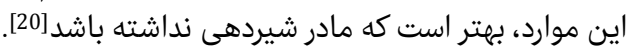

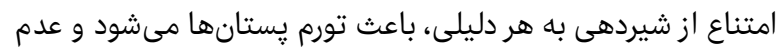

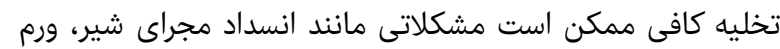

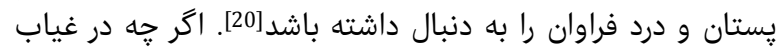

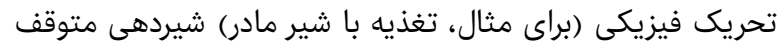

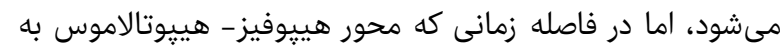

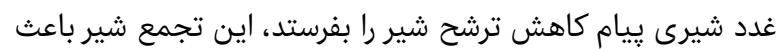

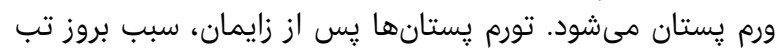

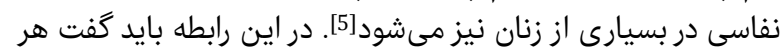

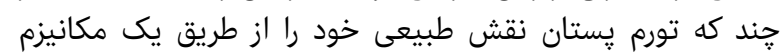

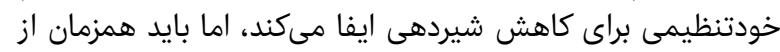

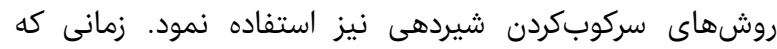

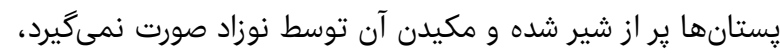

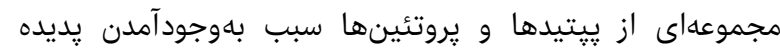

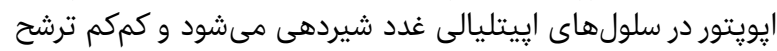

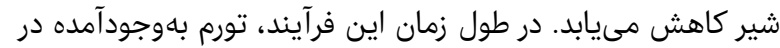

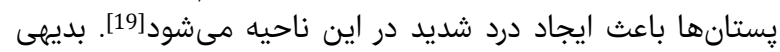

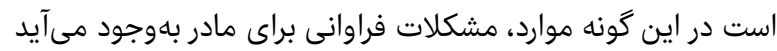

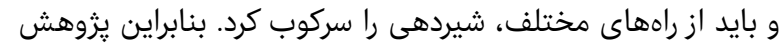

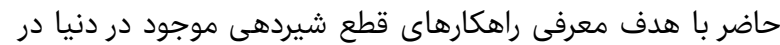

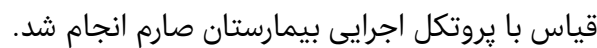

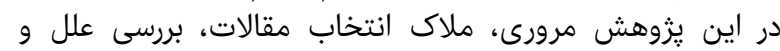

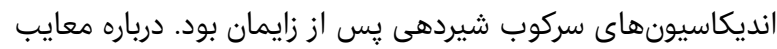

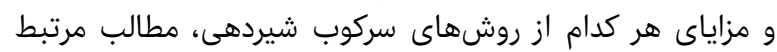

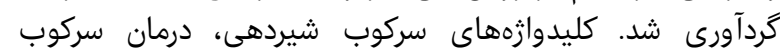

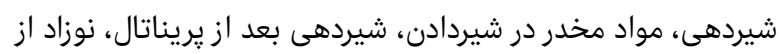

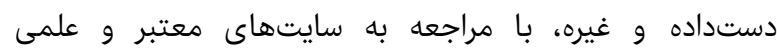
(Science Direct, Pubmed, Elsevier, Cochrane)
كاهش ضريب هوشى و مشكلات يادگيرى شدند [9]. حتى در بعضى

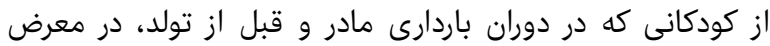

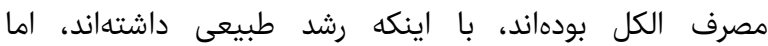

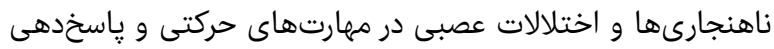

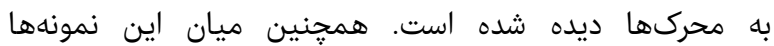

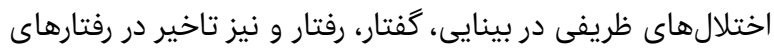

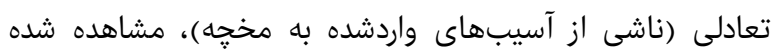

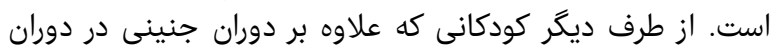

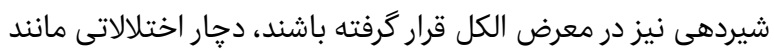
ADHD

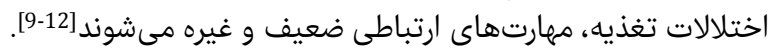

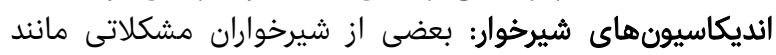

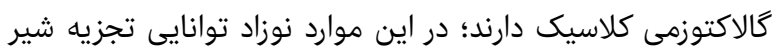

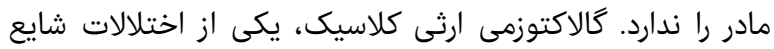

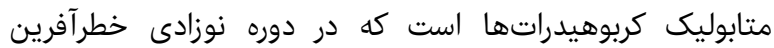

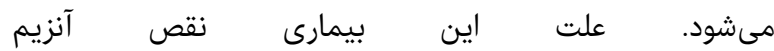

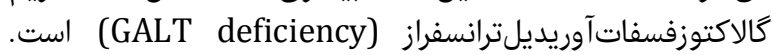

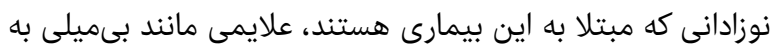

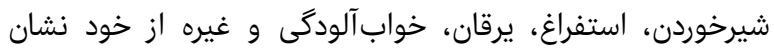

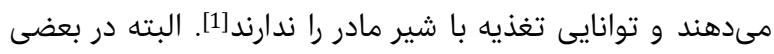

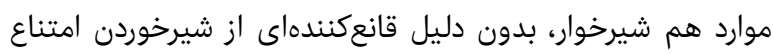
مىكند.

انتخاب شخصى مادر: در بسيارى از موارد، مادران بهدليل

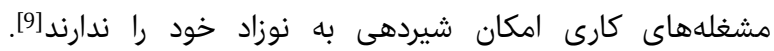

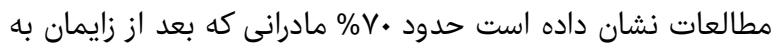

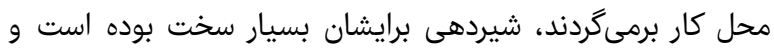

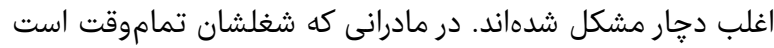

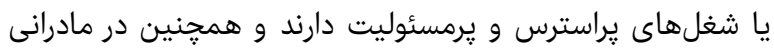

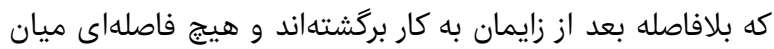
زايمان ايشان و شروع مجدد به كار وجود نداشته است، بـان احتمال

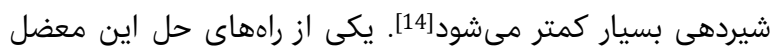

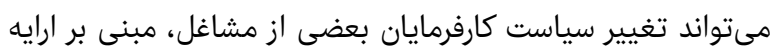

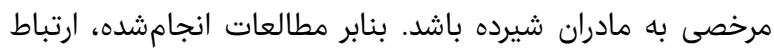

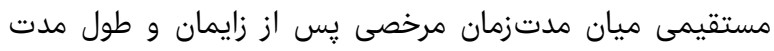

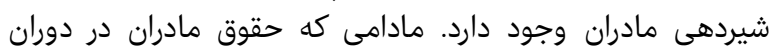

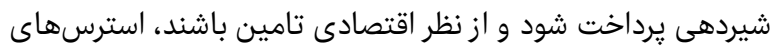

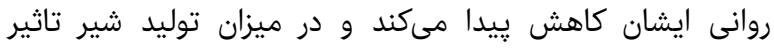

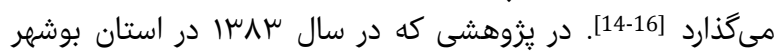

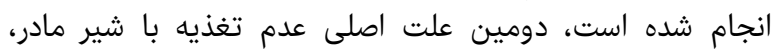

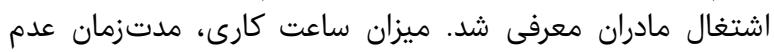

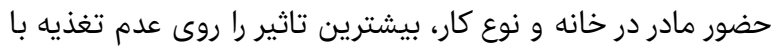
شير مادر داشت[15].

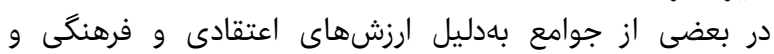

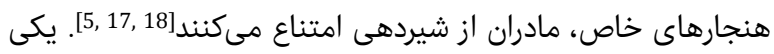

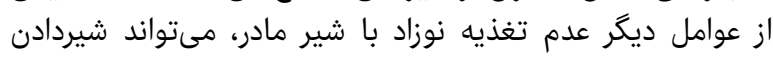

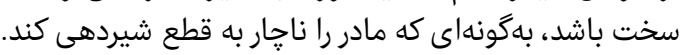

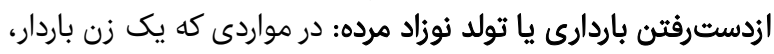

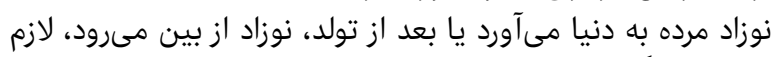

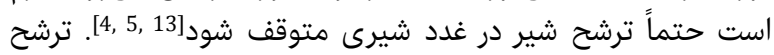

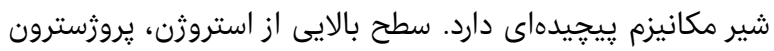

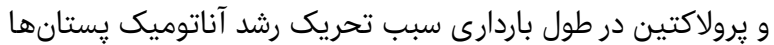

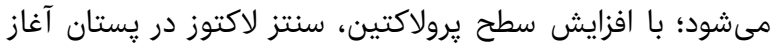


و تورم يستانها بهطور كامل، با استفاده از اين دارو برطرف

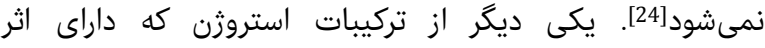

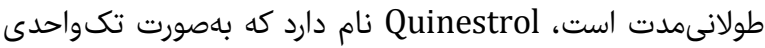
با دوز عميلىگرم، بلافاصله يس از زايت، زايمان تجويز مى شود[25]. اتينيل

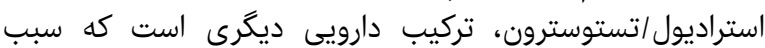

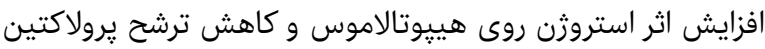

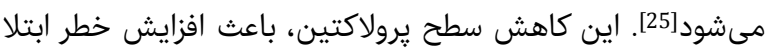

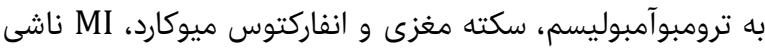

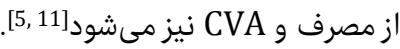

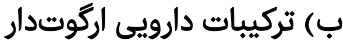

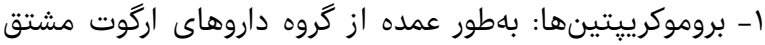

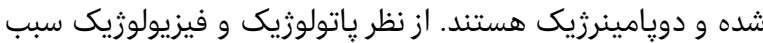

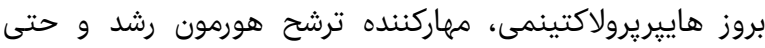
ضدياركينسون هستند [20].

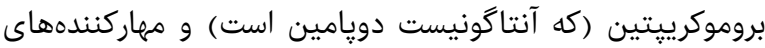

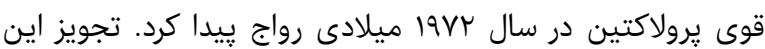

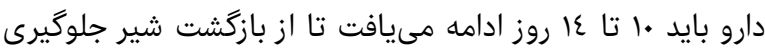

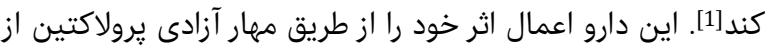

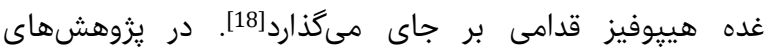

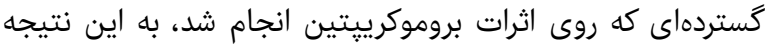

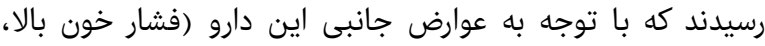

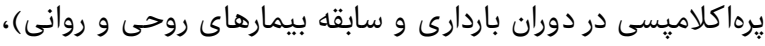

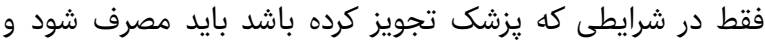

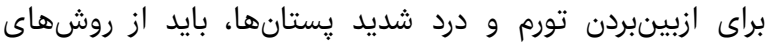

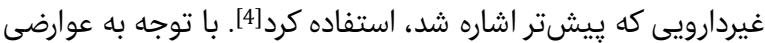

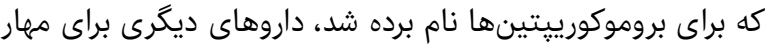

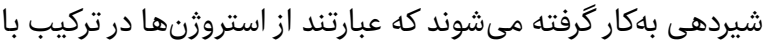

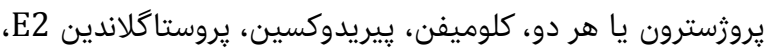

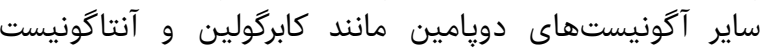

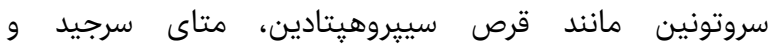
metergoline

يس از زايمان نشان دادهاند[5].

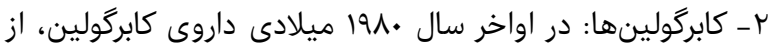

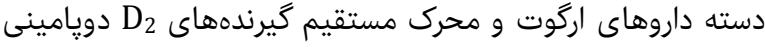

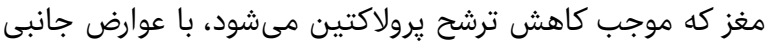

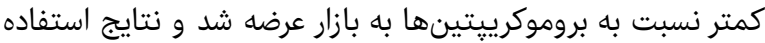

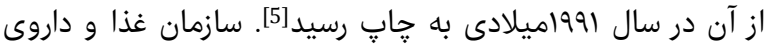

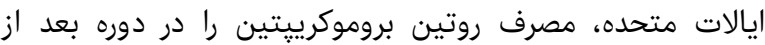

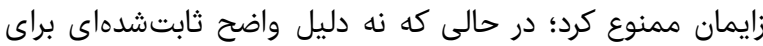

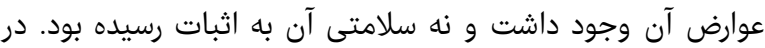

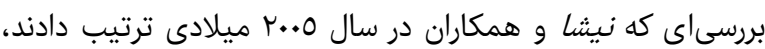

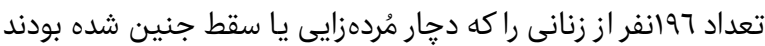

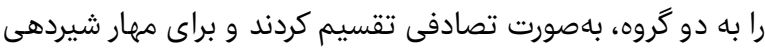

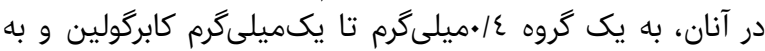

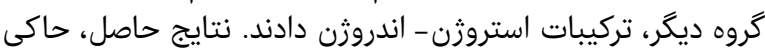

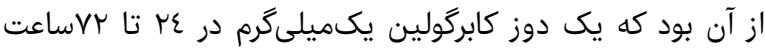

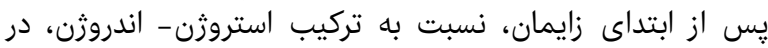

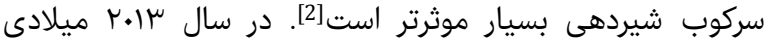

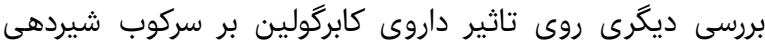

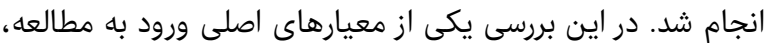

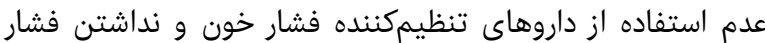

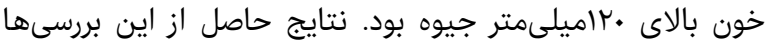
دوره ا، شماره ا، زمستان هوسر

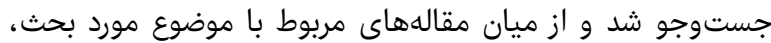

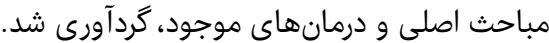

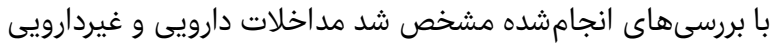

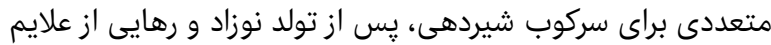

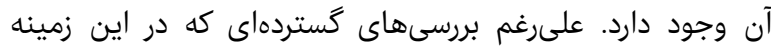

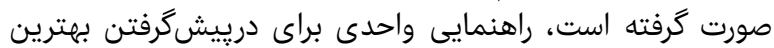

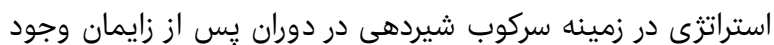

ندارد.

روشهاى غيردارويى و استفاده از طب مكمل: قرنهاست بشر براى

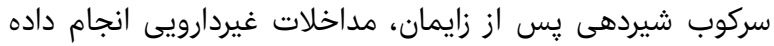

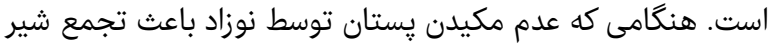
در آلوئولها مىشود و تحريك رفلكس ترشح فاكتورهاى مهاركنينده

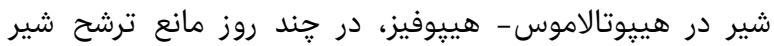

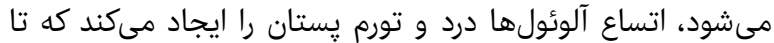

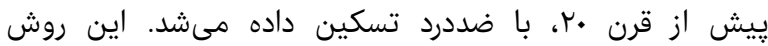

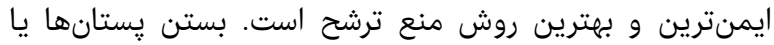

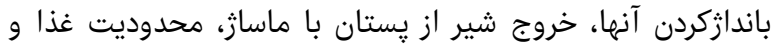

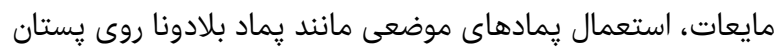

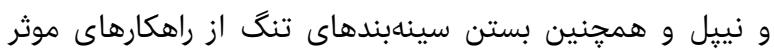

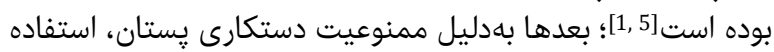

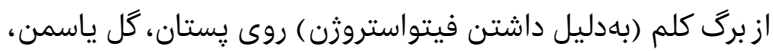

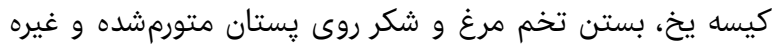

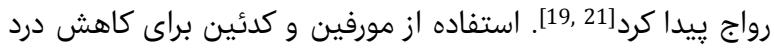

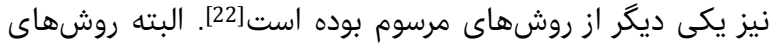

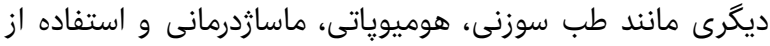

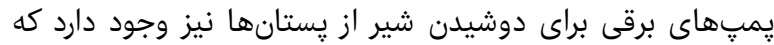

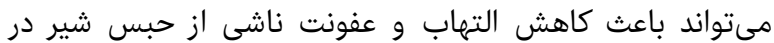

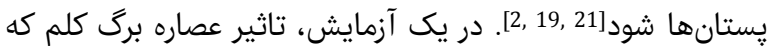

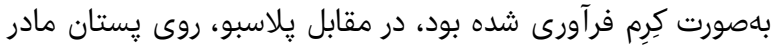

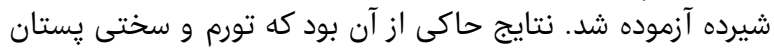

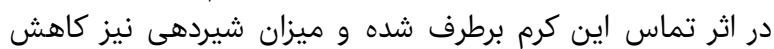

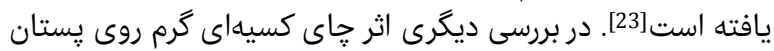

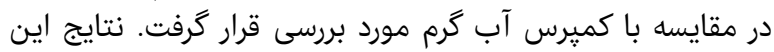

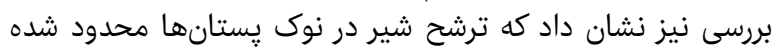

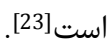

روشهاى دارويى: روشهاى دارويى زيادى براى مهار شيردهى

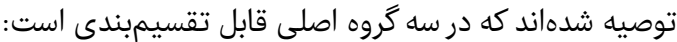
الف) تركيبات استروثن تاثير استروزن روى غيبات استرؤن يستانى كاملاً شناختهشده نيست، اما

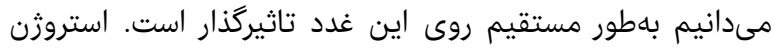

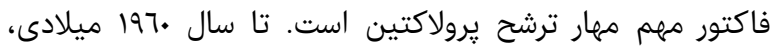

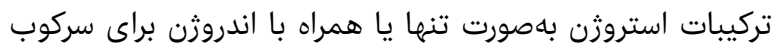

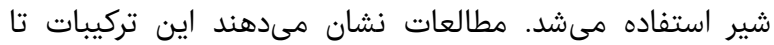

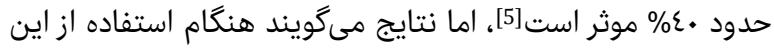

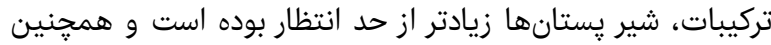

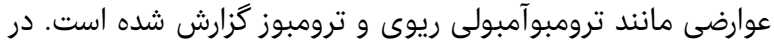

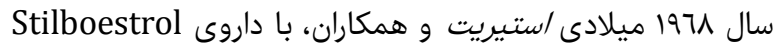

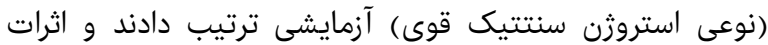

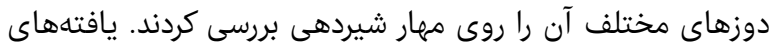

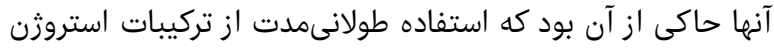

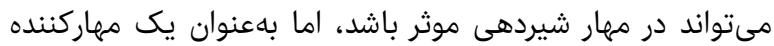

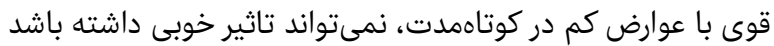


مجبور به قطع ترشح شير هستيم. با وجود بررسىهاى زيادى كهان كه در

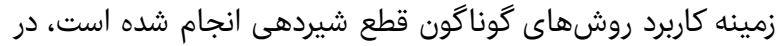

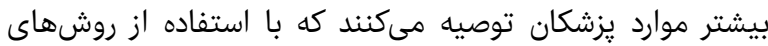

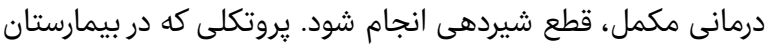

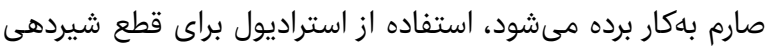

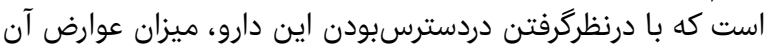

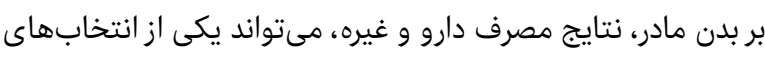

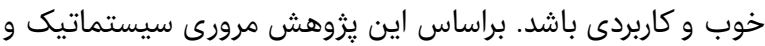

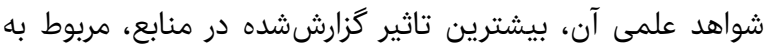

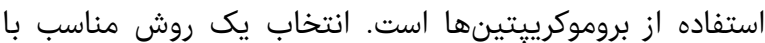

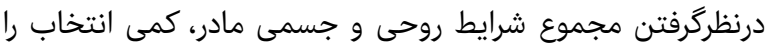

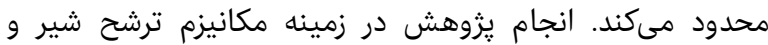

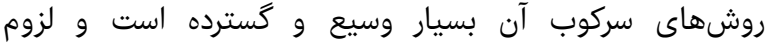

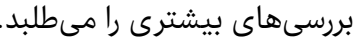

تشكر و قدردانى: موردى از سوى نويسندگان ذكر نشده است.

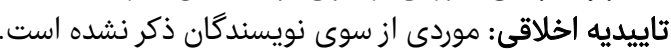

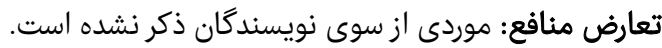

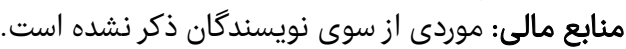

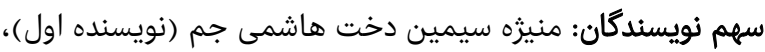

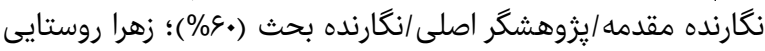

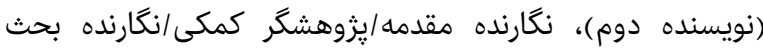

(\% K.)

منابع

1- AlSaad D, ElSalem S, Abdulrouf PV, Thomas B, Alsaad $\mathrm{T}$, Ahmed A, et al. A retrospective drug use evaluation of cabergoline for lactation inhibition at a tertiary care teaching hospital in Qatar. Ther Clin Risk Manag. 2016;12:155-60.

2- Nisha S, Uma S, Vineeta S. Role of newer drug cabergoline in lactation suppression as compared to estrogen-androgen combination. J Obstet Gynecol India. 2009;59(2):152-5.

3- Chaves RG, Lamounier JA. Breastfeeding and maternal medications. J Pediatria. 2004;80(5):S189-98.

4- Haghikia A, Podewski E, Berliner D, Sonnenschein K, Fischer D, Angermann CE, et al. Rationale and design of a randomized, controlled multicentre clinical trial to evaluate the effect of bromocriptine on left ventricular function in women with peripartum cardiomyopathy. Clin Res Cardiol. 2015;104(11):911-7.

5- Oladapo OT, Fawole B. Treatments for suppression of lactation. Cochrane Database Syst Rev. 2012;9:CD005937.

6- Sadeghi M, Ariyafar M, Maghsoudloo M. Clinical considerations during childbirth in drug dependent mothers, population health, family and schools. Tehran: Office of Prevention and Combating Substance Abuse; 2011. pp. 32-47. [Persian]

7- Javan R, Delbari A, Tabaraei Y, Hashemian M. A Study of the association between drug abuse and duration of exclusive breastfeeding in mothers in Sabzevar City, Iran. Qom Univ Med Sci J. 2014;8(3):55. [Persian]

8- Nowrouzi AL, Saberi Zafarghandi MB, Gilanipour M, Nasehi AA, Nickfarjam A, Jafari F, et al. Handbook on drug dependence management in pregnancy, childbirth, lactation and infancy. Tehran: Ministry of Health and Medical Education Press; 2015. pp. 159-60. [Persian] 9- Guelinckx I, Devlieger R, Vansant G. Alcohol during
نشان داد كه اين دارو براى مادرانى كه در دوره باردارى دجار برار

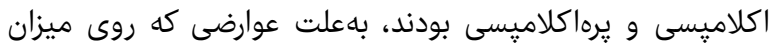

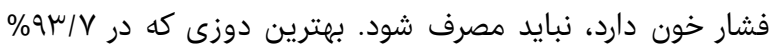

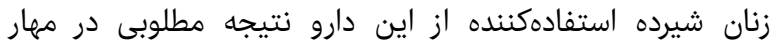

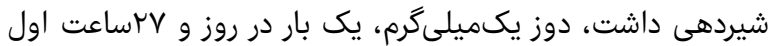

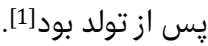

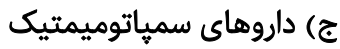
يزودوافدرين يك آمينسمياتوميمتيك است (ادريكين إنرزيك رسيتور)

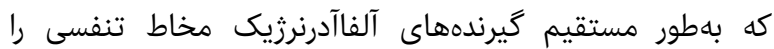

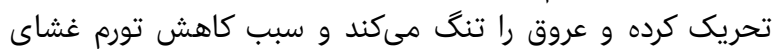

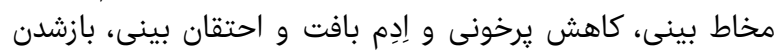

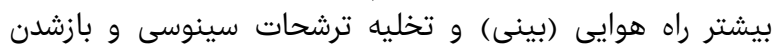

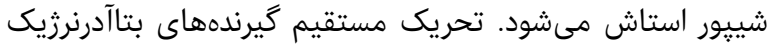

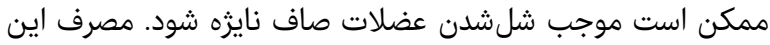

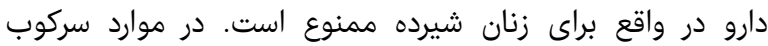

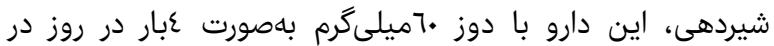

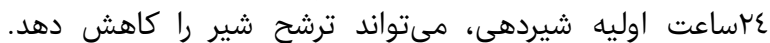

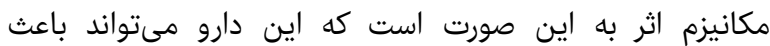

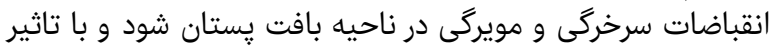

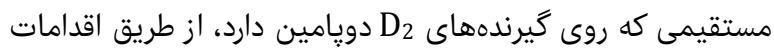

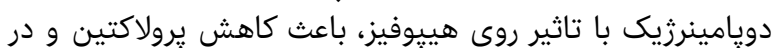
نهايت كاهش ترشح شير شود. موارد منع مصرف اين داروها ترونا

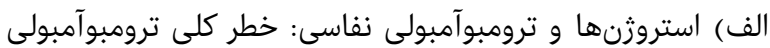

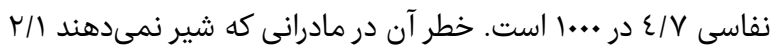

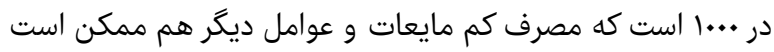

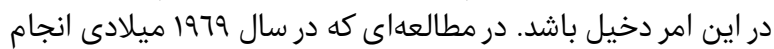

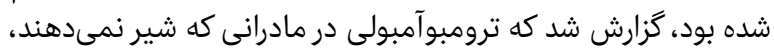

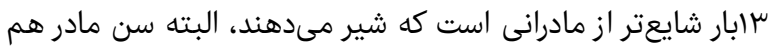

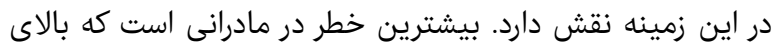

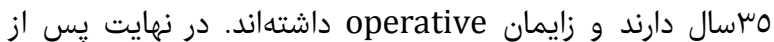

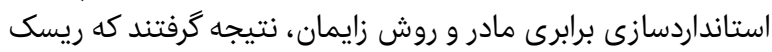

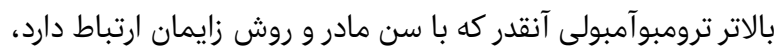

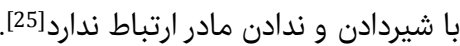

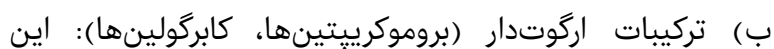

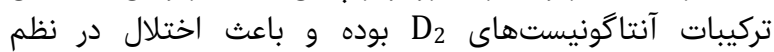

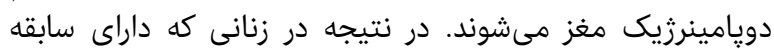

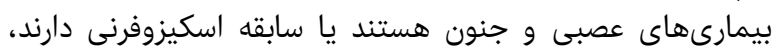

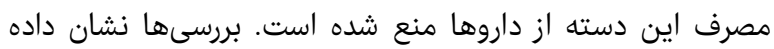

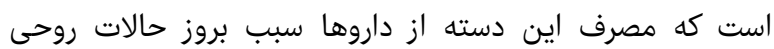

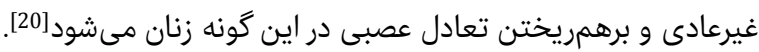

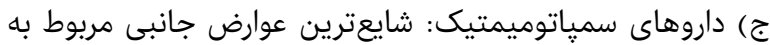

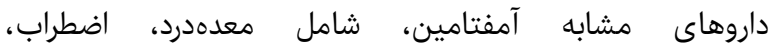

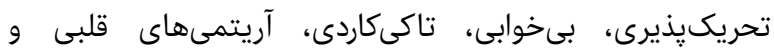

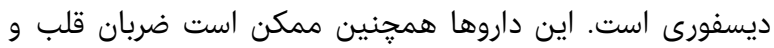

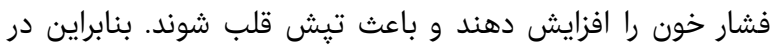
زنان شيرده و افرادى كه در دوران باردارى يرهاكلاميسى دئ داشتهاند

توصيه نمى شود.

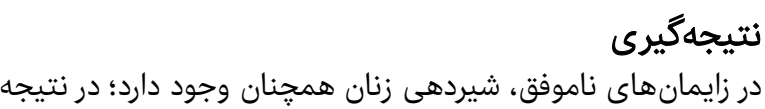

در زايمانهاى ناموفق، شيردهى زنان همجنان وجود دارد؛ در نتيجه 
17- Rahimzadeh M, Hosseini M, Mahmoodi M, Mohammad K. A survey on some effective factors on the duration of breastfeeding using survival analysis (Mazandaran province). J Semnan Univ Med Sci. 2007;8(3):161-70. [Persian]

18- Sereshti M, Nahidi F, Simbar M, Bakhtiari M, Zayeri F. An exploration of the maternal experiences of breast engorgement and milk leakage after perinatal loss. Glob J Health Sci. 2016;8(9):53876.

19- Cole M. Lactation after perinatal, neonatal, or infant loss. Clin Lactation. 2012;3(3):94-100.

20- Snellen M, Power J, Blankley G, Galbally M. Pharmacological lactation suppression with D2 receptor agonists and risk of postpartum psychosis: A systematic review. Aust N Z J Obstet Gynaecol. 2016;56(4):336-40.

21- McGuinness D, Coghlan B, Butler M. An exploration of the experiences of mothers as they suppress lactation following late miscarriage, stillbirth or neonatal death. which citty? évid Based Midwifery. 2014;12:65-70.

22- Spitz AM, Lee NC, Peterson HB. Treatment for lactation suppression: Little progress in one hundred years. Am j obstet gynecol. 1998;179(6):1485-90.

23- Anderson FW, Johnson CT. Complementary and alternative medicine in obstetrics. Int J Gynaecol Obstet. 2005;91(2):116-24.

24- Stirrat G, Anderson G, Grant 0. The effectiveness of stilboestrol in the suppression of postpartum lactation. Int J Obstet Gynaecol. 1968;75(3):313-5.

25- Llewellyn Jones D. Inhibition of lactation. Drugs. 1975;10(2):121-9. versus real intake. Arch Public Health. 2011;68(4):134.

10- Onu J, Oke B, Ozegbe P, Oyewale J. Effects of alcohol consumption during pregnancy and/or lactation on the morphology of thyroid gland in male Wistar rat offspring. Pak Vet J. 2011;31(4):357-9.

11- Smith L. Alcohol consumption during pregnancy and breast feeding in Canada is prevalent and not strongly associated with mental health status. Evid Based Nurs. 2017;20(2):44.

12- Jahani Moghaddam Z. Investigation of the damaging effects of alcohol consumption during lactation on cerebellum structure and balance of neonates [Dissertation]. Tehran: Shahid Beheshti University; 1998. [Persian]

13- Newman J. When breast-feeding is not contraindicated: Do you know when to stop breastfeeding? Can Fam Physician. 1991;37:969-75.

14- Dagher RK, McGovern PM, Schold JD, Randall XJ. Determinants of breastfeeding initiation and cessation among employed mothers: A prospective cohort study. BMC Pregnancy Childbirth. 2016;16:194.

15- Ghaed Mohamamdi Z, Zafarmand MH, Heydary G, Anaraki A, Dehghan A. Determination of effective factors in breast feeding continuity for infants less than 1 year old in urban area of Bushehr Province. Iran South Med J. 2004;7(1):79-87. [Persian]

16- Dun-Dery EJ, Laar AK. Exclusive breastfeeding among city-dwelling professional working mothers in Ghana. Int Breastfeed J. 2016;11:23. 